J. Austral. Math. Soc. 19 (Series A) (1975), 252-256.

\title{
THE RAMSEY NUMBER FOR STRIPES
}

\author{
E. J. COCKAYNE and P. J. LORIMER
}

(Received 5 April 1973; revised 5 February 1974)

Communicated by G. Szekeres

If $G_{1}, \cdots, G_{c}$ are graphs without loops or multiple edges there is a smallest integer $r\left(G_{1}, \cdots, G_{c}\right)$ such that if the edges of a complete graph $K_{n}$, with $n \geqq r\left(G_{1}, \cdots, G_{q}\right)$, are painted arbitrarily with $c$ colours the $i$ th coloured subgraph contains $G_{i}$ as a subgraph for at least one i. $r\left(G_{1}, \cdots, G_{c}\right)$ is called the Ramsey number of the graphs $G_{1}, \cdots, G_{c}$.

Ramsey graph theory was formulated in Cockayne (1970) and independently in Harary and Chratal (to appear). There has been considerable interest in the topic recently. Some properties of the numbers are mentioned in Cockayne (1972) and an extensive bibliography may be found in Harary (1972).

In this paper we determine $r\left(n_{1} P_{2}, \cdots, n_{c} P_{2}\right)$ where, in the notation of Harary (1969), $n P_{2}$ is the graph consisting of $2 n$ vertices and $n$ independent edges, called here a stripe.

By a circuit we shall mean a graph consisting of a finite number of vertices with each vertex joined to the next and with the last vertex joined to the first. By the complement of a subgraph we mean the vertex complement.

We shall prove

THEOREM. If $n_{1}, \cdots, n_{c}$ are positive integers and $n_{1}=\max \left(n_{1}, \cdots, n_{c}\right)$ then

$$
r\left(n_{1} P_{2}, \cdots, n_{c} P_{2}\right)=n_{1}+1+\sum_{i=1}^{c}\left(n_{i}-1\right) .
$$

This theorem has the following consequence.

COROLlARY. If a complete graph on $n$ vertices has its edges coloured by $c$ colours then it has a monochromatic subgraph isomorphic to $w_{2} P_{2}$ where $w$ is the largest integer not greater than

$$
\frac{n+c-1}{c+1}
$$


The corollary is proved by noticing that if $\frac{n+c-1}{c+1}$ is substituted for each $n_{i}$ in the expression

$$
n_{1}+1+\sum_{i=1}^{c}\left(n_{i}-1\right)
$$

the number $n$ is obtained.

Now we prove the theorem.

We show first that

$$
r\left(n_{1} P_{2}, \cdots, n_{c} P_{2}\right) \geqq n_{1}+1+\sum_{i=1}^{c}\left(n_{i}-1\right) .
$$

Consider a complete graph on

$$
n_{1}+\sum_{i=1}^{c}\left(n_{i}-1\right)
$$

vertices and partition the vertices into sets $V_{1}, \cdots, V_{c}$ where $V_{1}$ has $2 n_{1}-1$ members and each other $V_{i}$ has $n_{i}-1$. Paint with the first colour all edges which are incident with two vertices of $V_{1}$. For each $i=2, \cdots, c$ paint with the $i$ th colour the edges having two vertices in $V_{i}$ or one vertex in $V_{i}$ and one in $V_{j}$ where $j<i$. For each $i=1, \cdots, c$ the $i$ th coloured subgraph does not contain a subgraph isomorphic to $n_{i} P_{2}$ and so

$$
r\left(n_{1} P_{2}, \cdots, n_{c} P_{2}\right) \geqq n_{1}+1+\sum_{i=1}^{c}\left(n_{i}-1\right) .
$$

The rest of this paper is devoted to proving the opposite inequality, that is,

$$
r\left(n_{1} P_{2}, \cdots, n_{c} P_{2}\right) \leqq n_{1}+1+\sum_{i=1}^{c}\left(n_{i}-1\right) .
$$

Suppose that counterexamples exist; that is, there are positive integers $c$, $n_{1}, \cdots, n_{c}$ with $n_{1}=\max \left(n_{1}, \cdots, n_{c}\right)$ and a complete graph on at least

$$
n_{1}+1+\sum_{i=1}^{c}\left(n_{i}-1\right)
$$

vertices which does not have a subgraph isomorphic to $n_{i} P_{2}$ and coloured by the $i$ th colour for any $i=1, \cdots, c$. Among the counterexamples let $G$ be a minimal one in the following sense:

(1) $G$ is coloured with $c$ colours and no counterexample is coloured with less than $c$ colours.

(2) Among the counterexamples satisfying (1), $G$ is one having a minimal number of vertices. 
It is easily seen that these conditions imply that $G$ has exactly

$$
n_{1}+1+\sum_{i=1}^{c}\left(n_{i}-1\right)
$$

vertices, for any subgraph of $G$ having this number of vertices is also a counterexample and so, because of the minimal properties of $G$, it coincides with $G$.

The analysis now depends on the study of certain circuits and trees in $G$. These are the ones having no two adjacent edges of the same colour. If $C$ is one of these subgraphs which has $\alpha_{i}$ edges coloured by the $i$ th colour for each $i=1, \cdots, c$ then it has

$$
\varepsilon+\sum_{i=1}^{c} \alpha_{i}
$$

vertices where $\varepsilon=0$ if $C$ is a circuit and $\varepsilon=1$ if $C$ is a tree. For each $i$ the $\alpha_{i}$ edges coloured by the $i$ th colour form a subgraph of $C$ isomorphic to $\alpha_{i} P_{2}$. If $C^{\prime}$ is the complementary graph to $C$ in $G$ the number of vertices in $C^{\prime}$ is

$$
n_{1}+1+\sum_{i=1}^{c}\left(n_{i}-1\right)-\left(\varepsilon+\sum_{i=1}^{c} \alpha_{i}\right)=\left(n_{1}-\varepsilon\right)+1+\sum_{i=1}^{c}\left(n_{i}-\alpha_{i}-1\right) .
$$

Suppose that the numbers $n_{i}-\alpha_{i}, i=1, \cdots, c$ are $m_{1}, \cdots, m_{c}$ in decreasing order. If $C$ is a circuit, $\varepsilon=0$ and

$$
\begin{aligned}
n_{1}-\varepsilon & =n_{1} \\
& \geqq n_{i}-\alpha_{i} \text { for each } i=1, \cdots, c .
\end{aligned}
$$

If $C$ is a tree having the additional property that it has at least one edge coloured by each colour then $\alpha_{i}>0$ and

$$
\begin{aligned}
n_{1}-\varepsilon & =n_{1}-1 \\
& \geqq n_{i}-\alpha_{i} \text { for each } i=1, \cdots, c .
\end{aligned}
$$

In either case $n_{1}-\varepsilon \geqq m_{1}$ and the number of vertices in $C^{\prime}$ is at least

$$
m_{1}+1+\sum_{i=1}^{c}\left(m_{i}-1\right) \text {. }
$$

As $m_{1}=\max \left(m_{1}, \cdots, m_{c}\right)$ and $C^{\prime}$ is a proper subgraph of $G$ we deduce from the minimality of $G$ that $C^{\prime}$ has a subgraph isomorphic to $\left(n_{i}-\alpha_{i}\right) P_{2}$ coloured by the $i$ th colour for one of the $i=1, \cdots, c$. Combining this with the subgraph of $C$ isomorphic to $\alpha_{i} P_{2}$ and coloured by the same colour we obtain in $G$ a subgraph isomorphic to $n_{i} P_{2}$ and coloured by this colour. As this is assumed impossible we deduce that any circuit in $G$, and also any tree in $G$ having at least one edge of each colour, must have two adjacent edges of the same colour. 
Now consider the set of subgraphs of $G$ which are trees having no two adjacent edges of the same colour. As any edge is such a tree this set is not empty. In the set choose a tree, say $T$, having the maximum number of edges possible. We have shown above that not every colour occurs among the edges of $T$. Let $p q$ be an edge having a colour different from that of any edge of $T$. If both $p$ and $q$ are vertices of $T$ the edge $p q$ could be adjoined to a chain linking $p$ and $q$ in $T$ to form a circuit in which no two adjacent edges have the same colour. If exactly one of $p$ and $q$ is a vertex of $T$ then $p q$ can be adjoined to $T$ to form a tree having the same properties as $T$ but with one more vertex. In either case we have a contradiction so that neither $p$ nor $q$ are vertices of $T$.

We now work towards finding a contradiction to our assumption that a counterexample to the result exists. This is done by proving that the finite tree $T$ contains an infinite number of vertices. We prove that $T$ has an infinite sequence $r_{1}, r_{2}, \cdots$ of vertices with the properties:

(1) for each $i \geqq 1, r_{i} r_{i+1}$ is an edge of $T$,

(2) for each $i \geqq 2$ the vertices $r_{i-1}, r_{i}$, and $r_{i+1}$ are all different.

(3) For each $i \geqq 1$ the edges $r_{i} r_{i+1}$ and $r_{i} p$ have the same colour.

As $T$ is a tree it has no circuits so condition (2) here is sufficient to prove that the sequence $r_{1}, r_{2}, \cdots$ is infinite.

First let $r_{1}$ be any vertex of $T$ having valency one in $T$. Such vertices exist because $T$ is a tree. $r_{2}$ is defined by the condition that $r_{1} r_{2}$ is an edge of $T$. If the edge $r_{1} p$ is added to $T$ we get a tree $U$ having one more vertex than $T$. From the maximal property of $T, U$ must have two adjacent edges of the same colour. These can only be $r_{1} r_{2}$ and $r_{1} p$.

If the edge $r_{2} p$ is adjoined to $T$ we get a tree having one more vertex than $T$ and hence having two adjacent edges of the same colour. One of these edges must be $r_{2} p$ and then $r_{3}$ is defined by the property that $r_{2} r_{3}$ is an edge of $T$ having the same colour as $r_{2} p$. If $r_{3}=r_{1}$ we can form a tree from $T$ by deleting $r_{1} r_{2}$ and adding the edges $r_{2} p$ and $p q$. This tree has one more vertex than $T$ but has no adjacent edges of the same colour, a contradiction. Hence $r_{3} \neq r_{1}$.

Finally suppose that $i \geqq 2$, that $r_{1}, \cdots, r_{i+1}$ have been defined and that $r_{1}, \cdots, r_{i}$ have the required properties. We need to find an edge $r_{i+1} r_{i+2}$ of $T$ different from $r_{i} r_{i+1}$ having the same colour as $r_{i+1} p$.

Form a tree $U$ in $G$ by subtracting from $T$ any edge incident with $r_{i}$ and by adding the edges $p q, r_{i-1} p, r_{i+1} p$ and edges $q x$ whenever $r_{i} x$ is an edge of $T$ different from $r_{i} r_{i-1}$ or $r_{i} r_{i+1}$. As $U$ has one more vertex than $T$ two of its adjacent edges are of the same colour. As $T$ does not have two adjacent edges with this property the only possibilities are:

(a) $r_{i-1} p$ and an edge $y r_{i-1}$ of $T$ with $y \neq r_{i}$.

(b) $p q$ and $r_{i-1} p, r_{i+1} p$ or an edge $q x$ where $x$ is a vertex of $T$. 
(c) $r_{i+1} p$ with an edge $r_{i+1} r_{i+2}$ of $U$ with $r_{i+2}$ different from $r_{i}$.

(d) $q x$ and an edge $x y$ of $T$ where $x$ is different from $r_{i-1}, r_{i+1}$, and $r_{i} x, x y$ are edges of $T$.

We eliminate possibilities (a), (b) and (d).

If $y r_{i-1}$ is an edge of $T$ with $y \neq r_{i-1}$ the edges $y r_{i-1}$ and $r_{i-1} r_{i}$ being adjacent have different colours. By assumption the edges $r_{i-1} p$ and $r_{i-1} r_{i}$ have the same colour. Hence (a) cannot occur.

If (b) occurs we can adjoin to $T$ whichever edge $r_{i-1} p, r_{i+1} p$ or $q x$ has the same colour as $p q$ to obtain a tree having no two adjacent edges of the same colour yet having one more vertex than $T$. The impossibility of this excludes (b).

Suppose that (d) holds. Because of the properties of $T$ and $p q$, the edges $r_{i-1} r_{i}, r_{i} r_{i+1}$, and $p q$ all have different colours, say red, blue, and green respectively. By the induction assumption $r_{i-1} p$ is red and $r_{i} p$ is blue. Consider the circuit $r_{i} x q p$. It must have two adjacent edges of the same colour. As $r_{i} x$ and $r_{i} r_{i+1}$ are adjacent edges of $T, r_{i} x$ is not blue. As in the previous paragraph $p q$ and $q x$ have different colours. Hence $r_{i} x$ and $q x$ have the same colour. The assumption in (d) then gives two adjacent edges $r_{i} x$ and $x y$ of $T$ having the same colour. This eliminates (d).

Only the possibility (c) remains and this serves to define $r_{i+2}$ and establish the existence of the infinite sequence $r_{1}, r_{2}, \cdots$ in the finite tree $T$. This contradiction finishes the proof of the theorem.

\section{Acknowledgements}

The first author gratefully acknowledges the support of the Canadian National Research Council in the form of Grant NR.C. A7544. He also wishes to thank the Mathematical Institute, Oxford University and the Mathematics Department, University of Auckland for their excellent hospitality during visits in 1972 and 1973 respectively.

\section{References}

E. J. Cockayne (1970), 'An application of Ramseys Theorem', Can. Math. Bull. 13, 145-146.

E. J. Cockayne (1972), 'Colour Classes for r-graphs', Can. Math. Bull. 15, 349-354.

F. Harary (1969), Graph Theory (Addison-Wesley, Reading. Mass 1969).

F. Harary (1972), Recent results on generalised Ramsey Theory for Graphs. (To appear in proceedings of Kalmazoo Conf. on Graph Theory 1972).

F. Harary and V. Chratal (to appear), 'Generalised Ramsey Theory for Graphs', Bull. Amer. Math. Soc.

F. P. Ramsey (1930), 'On a problem of formal logic', Proc. London Math. Soc. 30, 264-286.

Department of Mathematics

University of Auckland

New Zealand
Department of Mathematics University of Victoria

British Columbia

Canada 